\title{
Various Fixed Point Theorems in Complex Valued $b$-Metric Spaces
}

\author{
Anil Kumar Dubey, ${ }^{1}$ Manjula Tripathi, ${ }^{2}$ and Ravi Prakash Dubey ${ }^{3}$ \\ ${ }^{1}$ Department of Mathematics, Bhilai Institute of Technology, Bhilai House, Durg, Chhattisgarh 491001, India \\ ${ }^{2}$ Department of Mathematics, U.P.U. Government Polytechnic, Durg, Chhattisgarh 491001, India \\ ${ }^{3}$ Department of Mathematics, Dr. C. V. Raman University, Kota, Bilaspur, Chhattisgarh 495113, India
}

Correspondence should be addressed to Anil Kumar Dubey; anilkumardby@rediffmail.com

Received 17 June 2016; Revised 24 September 2016; Accepted 11 October 2016

Academic Editor: Yurong Liu

Copyright (C) 2016 Anil Kumar Dubey et al. This is an open access article distributed under the Creative Commons Attribution License, which permits unrestricted use, distribution, and reproduction in any medium, provided the original work is properly cited.

We prove some common fixed point results for a pair of mappings which satisfy generalized contractive conditions with rational expressions having point-dependent control functions as coefficients in complex valued $b$-metric spaces. The results of this paper generalize and extend the several known results in complex valued $b$-metric spaces. Finally, examples are provided to verify the effectiveness and to usability of our main results.

\section{Introduction and Preliminaries}

The concept of complex valued metric space was introduced by Azam et al. [1], proving some fixed point results for mappings satisfying a rational inequality in complex valued metric spaces. Since then, several papers have dealt with fixed point theory in complex valued metric spaces (see [2-10] and references therein). Rao et al. [11] initiated the studying of fixed point results on complex valued $b$-metric spaces, which was more general than the complex valued metric spaces [1]. Following this paper, a number of authors have proved several fixed point results for various mapping satisfying a rational inequalities in the context of complex valued $b$-metric spaces (see [12-15]) and the related references therein.

Recently, Sintunavarat et al. [8,9], Sitthikul and Saejung [10], and Singh et al. [7] obtained common fixed point results by replacing the constant of contractive condition to control functions in complex valued metric spaces. In a continuation of $[7,10,14,16]$, in this paper, we establish some common fixed point results for a pair of mappings satisfying more general contractive conditions involving rational expressions having point-dependent control functions as coefficients in complex valued $b$-metric spaces.

Consistent with Rao et al. [11], the following definitions and results will be needed in the sequel.
Let $\mathbb{C}$ be the set of complex numbers and $z_{1}, z_{2} \in \mathbb{C}$. Define a partial order $\lesssim$ on $\mathbb{C}$ as follows:

$z_{1} \preccurlyeq z_{2}$ if and only if $\operatorname{Re}\left(z_{1}\right) \leq \operatorname{Re}\left(z_{2}\right), \operatorname{Im}\left(z_{1}\right) \leq \operatorname{Im}\left(z_{2}\right)$.

Consequently, one can infer that $z_{1} \precsim z_{2}$ if one of the following conditions is satisfied:

(i) $\operatorname{Re}\left(z_{1}\right)=\operatorname{Re}\left(z_{2}\right), \operatorname{Im}\left(z_{1}\right)<\operatorname{Im}\left(z_{2}\right)$.

(ii) $\operatorname{Re}\left(z_{1}\right)<\operatorname{Re}\left(z_{2}\right), \operatorname{Im}\left(z_{1}\right)=\operatorname{Im}\left(z_{2}\right)$.

(iii) $\operatorname{Re}\left(z_{1}\right)<\operatorname{Re}\left(z_{2}\right), \operatorname{Im}\left(z_{1}\right)<\operatorname{Im}\left(z_{2}\right)$.

(iv) $\operatorname{Re}\left(z_{1}\right)=\operatorname{Re}\left(z_{2}\right), \operatorname{Im}\left(z_{1}\right)=\operatorname{Im}\left(z_{2}\right)$.

In particular, we write $z_{1} \preccurlyeq z_{2}$ if $z_{1} \neq z_{2}$ and one of (i), (ii), and (iii) is satisfied and we write $z_{1} \prec z_{2}$ if only (iii) is satisfied. Notice that
(a) if $0 \precsim z_{1} \precsim z_{2}$, then $\left|z_{1}\right|<\left|z_{2}\right|$;
(b) if $z_{1} \preccurlyeq z_{2}$ and $z_{2} \prec z_{3}$, then $z_{1} \prec z_{3}$;
(c) if $a, b \in \mathbb{R}$ and $a \leq b$, then $a z \lesssim b z$ for all $z \in \mathbb{C}_{+}$.

The following definition is recently introduced by Rao et al. [11].

Definition 1 (see [11]). Let $X$ be a nonempty set and let $s \geq$ 1 be a given real number. A function $d: X \times X \rightarrow \mathbb{C}$ is 
called a complex valued $b$-metric on $X$ if for all $x, y, z \in X$ the following conditions are satisfied:

(i) $0 \precsim d(x, y)$ and $d(x, y)=0$ if and only if $x=y$.

(ii) $d(x, y)=d(y, x)$.

(iii) $d(x, y) \lesssim s[d(x, z)+d(z, y)]$.

The pair $(X, d)$ is called a complex valued $b$-metric space.

Example 2 (see [11]). If $X=[0,1]$, define a mapping $d: X \times$ $X \rightarrow \mathbb{C}$ by $d(x, y)=|x-y|^{2}+i|x-y|^{2}$, for all $x, y \in X$. Then, $(X, d)$ is complex valued $b$-metric space with $s=2$.

Definition 3 (see [11]). Let $(X, d)$ be a complex valued $b$ metric space.

(i) A point $x \in X$ is called interior point of a set $A \subseteq X$ whenever there exists $0 \prec r \in \mathbb{C}$ such that $B(x, r)=$ $\{y \in X: d(x, y) \prec r\} \subseteq A$.

(ii) A point $x \in X$ is called a limit point of a set $A$ whenever for every $0 \prec r \in \mathbb{C}, B(x, r) \cap(A-\{x\}) \neq \phi$.

(iii) A subset $A \subseteq X$ is called an open set whenever each element of $A$ is an interior point of $A$.

(iv) A subset $A \subseteq X$ is called closed set whenever each limit point of $A$ belongs to $A$.

(v) The family $F=\{B(x, r): x \in X$ and $0 \prec r\}$ is a subbasis for a Hausdorff topology $\tau$ on $X$.

Definition 4 (see [11]). Let $(X, d)$ be a complex valued $b$ metric space, and let $\left\{x_{n}\right\}$ be a sequence in $X$ and $x \in X$.

(i) If for every $c \in \mathbb{C}$, with $0 \prec c$, there is $N \in \mathbb{N}$ such that for all $n>N, d\left(x_{n}, x\right) \prec c$, then $\left\{x_{n}\right\}$ is said to be convergent and converges to $x$. We denote this by $\lim _{n \rightarrow \infty} x_{n}=x$ or $\left\{x_{n}\right\} \rightarrow x$ as $n \rightarrow \infty$.

(ii) If for every $c \in \mathbb{C}$, with $0 \prec c$ there is $N \in \mathbb{N}$ such that for all $n>N, d\left(x_{n}, x_{n+m}\right) \prec c$, where $m \in \mathbb{N}$, then $\left\{x_{n}\right\}$ is said to be a Cauchy sequence.

(iii) If every Cauchy sequence in $X$ is convergent in $X$, then $(X, d)$ is said to be a complete complex valued $b$-metric space.

Lemma 5 (see [11]). Let $(X, d)$ be a complex valued b-metric space and let $\left\{x_{n}\right\}$ be a sequence in $X$. Then, $\left\{x_{n}\right\}$ converges to $x$ if and only if $\left|d\left(x_{n}, x\right)\right| \rightarrow 0$ as $n \rightarrow \infty$.

Lemma 6 (see [11]). Let $(X, d)$ be a complex valued b-metric space and let $\left\{x_{n}\right\}$ be a sequence in $X$. Then, $\left\{x_{n}\right\}$ is Cauchy sequence if and only if $\left|d\left(x_{n}, x_{n+m}\right)\right| \rightarrow 0$ as $n \rightarrow \infty$, where $m \in \mathbb{N}$.

\section{Main Result}

Throughout this paper, let $(X, d)$ be a complete complex valued $b$-metric space and $S, T: X \rightarrow X$ be mappings. In our results, we will use the following family of functions.

Let $(X, d)$ be a complete complex valued $b$-metric space with the coefficient $s \geq 1$ and let $S, T: X \rightarrow X$ be mappings.
Let $\Psi$ be the family of all functions $\psi: X \times X \times X \rightarrow[0,1)$ such that for all $x, y \in X$ and for fixed $a \in X$,

(F1) $\psi(T S x, y, a) \leq \psi(x, y, a)$

(F2) $\psi(x, S T y, a) \leq \psi(x, y, a)$.

We start this section with the following observation.

Proposition 7. Let $(X, d)$ be a complex valued b-metric space and let $S, T: X \rightarrow X$ be mappings. Let $x_{0} \in X$ and define the sequence $\left\{x_{n}\right\}$ by

$$
\begin{aligned}
& x_{2 n+1}=S x_{2 n}, \\
& x_{2 n+2}=T x_{2 n+1}, \\
& \quad \forall n=0,1,2, \ldots
\end{aligned}
$$

Assume that there exists a mapping $\alpha \in \Psi$ for all $x, y \in X$ and for a fixed element $a \in X$ and $n=0,1,2, \ldots$ Then, $\alpha\left(x_{2 n}, y, a\right) \leq \alpha\left(x_{0}, y, a\right)$ and $\alpha\left(x, x_{2 n+1}, a\right) \leq \alpha\left(x, x_{1}, a\right)$.

Proof. Let $x, y \in X$ and $n=0,1,2, \ldots$. Then, we have

$$
\begin{aligned}
\alpha\left(x_{2 n}, y, a\right) & =\alpha\left(T x_{2 n-1}, y, a\right)=\alpha\left(T S x_{2 n-2}, y, a\right) \\
& \leq \alpha\left(x_{2 n-2}, y, a\right)=\alpha\left(T S x_{2 n-4}, y, a\right) \leq \cdots \\
& \leq \alpha\left(x_{0}, y, a\right)
\end{aligned}
$$

Similarly, we have

$$
\begin{aligned}
\alpha\left(x, x_{2 n+1}, a\right) & =\alpha\left(x, S x_{2 n}, a\right)=\alpha\left(x, S T x_{2 n-1}, a\right) \\
& \leq \alpha\left(x, x_{2 n-1}, a\right)=\alpha\left(x, S T x_{2 n-3}, a\right) \\
& \leq \cdots \leq \alpha\left(x, x_{1}, a\right) .
\end{aligned}
$$

Lemma 8 (see [10]). Let $\left\{x_{n}\right\}$ be a sequence in $X$ and $h \in[0,1)$. If $a_{n}=\left|d\left(x_{n}, x_{n+1}\right)\right|$ satisfies $a_{n} \leq h a_{n-1}$, for all $n \in \mathbb{N}$, then $\left\{x_{n}\right\}$ is a Cauchy sequence.

Now, we proceed to establish common fixed point theorems for the general contraction conditions in complex valued $b$-metric space.

Theorem 9. Let $(X, d)$ be a complete complex valued b-metric space with the coefficient $s \geq 1$ and let $S, T: X \rightarrow X$ be mappings. If there exist mappings $\alpha, \beta, \gamma, \delta \in \Psi$ such that for all $x, y \in X$ and for fixed $a \in X$,

$$
\begin{aligned}
\text { (a) } & \alpha(x, y, a)+\beta(x, y, a)+2 \gamma(x, y, a)+2 s \delta(x, y, a) \\
<< & 1
\end{aligned}
$$


(b) $d(S x, T y)$

$$
\begin{aligned}
& \precsim \alpha(x, y, a) d(x, y) \\
& \quad+\frac{\beta(x, y, a) d(y, T y) d(x, S x)}{1+d(x, y)} \\
& \quad+\gamma(x, y, a)[d(x, S x)+d(y, T y)] \\
& \quad+\delta(x, y, a)[d(x, T y)+d(y, S x)] .
\end{aligned}
$$

Then, $S$ and $T$ have a unique common fixed point.

Proof. Let $x, y \in X$, from (5) we have

$$
\begin{aligned}
d(S x, T S x) \lesssim & \alpha(x, S x, a) d(x, S x) \\
& +\frac{\beta(x, S x, a) d(S x, T S x) d(x, S x)}{1+d(x, S x)} \\
& +\gamma(x, S x, a)[d(x, S x)+d(S x, T S x)] \\
& +\delta(x, S x, a)[d(x, T S x)+d(S x, S x)] \\
\lesssim & \alpha(x, S x, a) d(x, S x) \\
& +\frac{\beta(x, S x, a) d(S x, T S x) d(x, S x)}{1+d(x, S x)} \\
& +\gamma(x, S x, a)[d(x, S x)+d(S x, T S x)] \\
& +s \delta(x, S x, a)[d(x, S x)+d(S x, T S x)]
\end{aligned}
$$

which implies that

$$
\begin{aligned}
& |d(S x, T S x)| \\
& \leq \alpha(x, S x, a)|d(x, S x)| \\
& \quad+\frac{\beta(x, S x, a)|d(S x, T S x)||d(x, S x)|}{|1+d(x, S x)|} \\
& \quad+\gamma(x, S x, a)|d(x, S x)+d(S x, T S x)| \\
& \quad+s \delta(x, S x, a)|d(x, S x)+d(S x, T S x)| .
\end{aligned}
$$

Since $|1+d(x, S x)| \geq|d(x, S x)|$,

$$
\begin{aligned}
& |d(S x, T S x)| \\
& \leq \alpha(x, S x, a)|d(x, S x)|+\beta(x, S x, a)|d(S x, T S x)| \\
& \quad+\gamma(x, S x, a)|d(x, S x)+d(S x, T S x)| \\
& \quad+s \delta(x, S x, a)|d(x, S x)+d(S x, T S x)| .
\end{aligned}
$$

In a similar way, by setting $x=T y$ in (5), we have

$$
\begin{aligned}
& |d(S T y, T y)| \\
& \leq \alpha(T y, y, a)|d(T y, y)| \\
& \quad+\beta(T y, y, a)|d(T y, S T y)| \\
& \quad+\gamma(T y, y, a)|d(y, T y)+d(T y, S T y)| \\
& \quad+s \delta(T y, y, a)|d(y, T y)+d(T y, S T y)| .
\end{aligned}
$$

Let $x_{0} \in X$ and the sequence $\left\{x_{n}\right\}$ be defined by (1). We show that $\left\{x_{n}\right\}$ is a Cauchy sequence. From Proposition 7 and for all $K=0,1,2, \ldots$, we obtain

$$
\begin{aligned}
& \left|d\left(x_{2 K+1}, x_{2 K}\right)\right|=\left|d\left(S T x_{2 K-1}, T x_{2 K-1}\right)\right| \\
& \quad \leq \alpha\left(x_{2 K}, x_{2 K-1}, a\right)\left|d\left(x_{2 K}, x_{2 K-1}\right)\right| \\
& +\beta\left(x_{2 K}, x_{2 K-1}, a\right)\left|d\left(x_{2 K+1}, x_{2 K}\right)\right| \\
& \quad+\gamma\left(x_{2 K}, x_{2 K-1}, a\right) \\
& \quad \cdot\left[\left|d\left(x_{2 K+1}, x_{2 K}\right)+d\left(x_{2 K}, x_{2 K-1}\right)\right|\right] \\
& +s \delta\left(x_{2 K}, x_{2 K-1}, a\right) \\
& \quad \cdot\left[\left|d\left(x_{2 K+1}, x_{2 K}\right)+d\left(x_{2 K}, x_{2 K-1}\right)\right|\right] \\
& \leq \alpha\left(x_{0}, x_{1}, a\right)\left|d\left(x_{2 K-1}, x_{2 K}\right)\right|+\beta\left(x_{0}, x_{1}, a\right) \\
& \quad \cdot\left|d\left(x_{2 K}, x_{2 K+1}\right)\right|+\gamma\left(x_{0}, x_{1}, a\right) \\
& \quad \cdot\left[\left|d\left(x_{2 K-1}, x_{2 K}\right)+d\left(x_{2 K}, x_{2 K+1}\right)\right|\right] \\
& \quad+s \delta\left(x_{0}, x_{1}, a\right)\left[\left|d\left(x_{2 K-1}, x_{2 K}\right)+d\left(x_{2 K}, x_{2 K+1}\right)\right|\right]
\end{aligned}
$$

which yields that

$$
\begin{aligned}
& \left|d\left(x_{2 K+1}, x_{2 K}\right)\right| \\
& \quad \leq \frac{\alpha\left(x_{0}, x_{1}, a\right)+\gamma\left(x_{0}, x_{1}, a\right)+s \delta\left(x_{0}, x_{1}, a\right)}{1-\beta\left(x_{0}, x_{1}, a\right)-\gamma\left(x_{0}, x_{1}, a\right)-s \delta\left(x_{0}, x_{1}, a\right)} \mid d\left(x_{2 K-1},\right. \\
& \left.\quad x_{2 K}\right) \mid .
\end{aligned}
$$

Similarly, one can obtain

$$
\begin{aligned}
& \left|d\left(x_{2 K+2}, x_{2 K+1}\right)\right| \\
& \quad \leq \frac{\alpha\left(x_{0}, x_{1}, a\right)+\gamma\left(x_{0}, x_{1}, a\right)+s \delta\left(x_{0}, x_{1}, a\right)}{1-\beta\left(x_{0}, x_{1}, a\right)-\gamma\left(x_{0}, x_{1}, a\right)-s \delta\left(x_{0}, x_{1}, a\right)} \mid d\left(x_{2 K},\right.
\end{aligned}
$$

$\left.x_{2 K+1}\right) \mid$.

Let $\mu=\left(\alpha\left(x_{0}, x_{1}, a\right)+\gamma\left(x_{0}, x_{1}, a\right)+s \delta\left(x_{0}, x_{1}, a\right)\right) /(1-$ $\left.\beta\left(x_{0}, x_{1}, a\right)-\gamma\left(x_{0}, x_{1}, a\right)-s \delta\left(x_{0}, x_{1}, a\right)\right)<1$.

Since $\alpha\left(x_{0}, x_{1}, a\right)+\beta\left(x_{0}, x_{1}, a\right)+2 \gamma\left(x_{0}, x_{1}, a\right)+$ $2 s \delta\left(x_{0}, x_{1}, a\right)<1$, thus we have $\left|d\left(x_{2 K+2}, x_{2 K+1}\right)\right| \leq$ $\mu\left|d\left(x_{2 K}, x_{2 K+1}\right)\right|$ and $\left|d\left(x_{2 K+1}, x_{2 K}\right)\right| \leq \mu\left|d\left(x_{2 K-1}, x_{2 K}\right)\right|$, or in fact

$$
\left|d\left(x_{n+1}, x_{n+2}\right)\right| \leq \mu\left|d\left(x_{n}, x_{n+1}\right)\right| \text {. }
$$

Thus, by Lemma 8 we get that this sequence is Cauchy sequence in $(X, d)$. Since $X$ is complete, there exists some $u \in X$ such that $x_{n} \rightarrow u$ as $n \rightarrow \infty$. Let, on contrary, $u \neq S u$; then

$$
|d(u, S u)|>0 \text {. }
$$


So by using the triangular inequality and (5), we get

$$
\begin{aligned}
d(u, S u) & s d\left(u, x_{2 n+2}\right)+s d\left(x_{2 n+2}, S u\right) \\
= & s d\left(u, x_{2 n+2}\right)+s d\left(T x_{2 n+1}, S u\right) \\
\lesssim & s d\left(u, x_{2 n+2}\right)+s \alpha\left(u, x_{2 n+1}, a\right) d\left(u, x_{2 n+1}\right) \\
& +\frac{s \beta\left(u, x_{2 n+1}, a\right) d\left(x_{2 n+1}, T x_{2 n+1}\right) d(u, S u)}{1+d\left(u, x_{2 n+1}\right)} \\
& +s \gamma\left(u, x_{2 n+1}, a\right)\left[d(u, S u)+d\left(x_{2 n+1}, T x_{2 n+1}\right)\right] \\
& +s \delta\left(u, x_{2 n+1}, a\right)\left[d\left(u, T x_{2 n+1}\right)+d\left(x_{2 n+1}, S u\right)\right] \\
\lesssim & s d\left(u, x_{2 n+2}\right)+s \alpha\left(u, x_{1}, a\right) d\left(u, x_{2 n+1}\right) \\
& +\frac{s \beta\left(u, x_{1}, a\right) d\left(x_{2 n+1}, x_{2 n+2}\right) d(u, S u)}{1+d\left(u, x_{2 n+1}\right)} \\
& +s \gamma\left(u, x_{1}, a\right)\left[d(u, S u)+d\left(x_{2 n+1}, x_{2 n+2}\right)\right] \\
& +s \delta\left(u, x_{1}, a\right)\left[d\left(u, x_{2 n+2}\right)+d\left(x_{2 n+1}, S u\right)\right] .
\end{aligned}
$$

This implies that

$$
\begin{aligned}
|d(u, S u)| & \\
\leq & s\left|d\left(u, x_{2 n+2}\right)\right|+s \alpha\left(u, x_{1}, a\right)\left|d\left(u, x_{2 n+1}\right)\right| \\
& +\frac{s \beta\left(u, x_{1}, a\right)\left|d\left(x_{2 n+1}, x_{2 n+2}\right)\right||d(u, S u)|}{\left|1+d\left(u, x_{2 n+1}\right)\right|} \\
& +s \gamma\left(u, x_{1}, a\right)\left|d(u, S u)+d\left(x_{2 n+1}, x_{2 n+2}\right)\right| \\
& +s \delta\left(u, x_{1}, a\right)\left|d\left(u, x_{2 n+2}\right)+d(u, S u)\right| .
\end{aligned}
$$

Letting $n \rightarrow \infty$, it follows that

$$
\begin{gathered}
|d(u, S u)| \leq s \gamma\left(u, x_{1}, a\right)|d(u, S u)|+s \delta\left(u, x_{1}, a\right) \\
\cdot|d(u, S u)|=s\left[\gamma\left(u, x_{1}, a\right)+\delta\left(u, x_{1}, a\right)\right] \\
\cdot|d(u, S u)| \leq s\left[\alpha\left(u, x_{1}, a\right)+\beta\left(u, x_{1}, a\right)\right. \\
\left.+2 \gamma\left(u, x_{1}, a\right)+2 \delta\left(u, x_{1}, a\right)\right]|d(u, S u)| \\
\quad<|d(u, S u)|
\end{gathered}
$$

a contradiction, and so $|d(u, S u)|=0$; that is, $u=S u$. It follows similarly that $u=T u$. This implies that $u$ is a common fixed point of $S$ and $T$.

We now prove that this $u$ is unique:

$$
\begin{aligned}
d\left(u, u^{*}\right)= & d\left(S u, T u^{*}\right) \\
\precsim & \alpha\left(u, u^{*}, a\right) d\left(u, u^{*}\right) \\
& +\frac{\beta\left(u, u^{*}, a\right) d\left(u^{*}, T u^{*}\right) d(u, S u)}{1+d\left(u, u^{*}\right)} \\
& +\gamma\left(u, u^{*} a\right)\left[d(u, S u)+d\left(u^{*}, T u^{*}\right)\right] \\
& +\delta\left(u, u^{*}, a\right)\left[d\left(u, T u^{*}\right)+d\left(u^{*}, S u\right)\right] \\
\precsim & {\left[\alpha\left(u, u^{*}, a\right)+2 \delta\left(u, u^{*} a\right)\right] d\left(u, u^{*}\right) . }
\end{aligned}
$$

Therefore, we have

$$
\left|d\left(u, u^{*}\right)\right| \leq\left[\alpha\left(u, u^{*}, a\right)+2 \delta\left(u, u^{*}, a\right)\right]\left|d\left(u, u^{*}\right)\right| .
$$

Since $\alpha\left(u, u^{*}, a\right)+2 \delta\left(u, u^{*}, a\right)<1$, we have $\left|d\left(u, u^{*}\right)\right|=0$.

Thus, $u=u^{*}$, which proves the uniqueness of common fixed point in $X$. This concludes the theorem.

Remark 10. If we replace $\alpha, \beta: X \times X \times X \rightarrow[0,1)$ by $\Lambda, E:$ $X \rightarrow[0,1)$, with $\alpha(x, y, a)=\Lambda(x)$ and $\beta(x, y, a)=E(x)$ for all $x, y \in X$ and so $s \Lambda(x)+E(x)<1$, then we get the result of Theorem 3.1 of Sintunavarat and Kumam [8] (complex valued $b$-metric space version).

Remark 11. If we set mappings $\alpha, \beta: X \times X \times X \rightarrow[0,1)$ as $\alpha(x, y, a)=\alpha^{\prime}$ and $\beta(x, y, a)=\beta^{\prime}$, where $\alpha^{\prime}, \beta^{\prime} \in[0,1)$ such that $s \alpha^{\prime}+\beta^{\prime}<1$ and for all $x, y \in X$, we get Theorem 4 of Azam et al. [1] (complex valued $b$-metric space version).

Next theorem is presented for single mapping satisfying slightly different conditions.

Theorem 12. Let $(X, d)$ be a complete complex valued $b$-metric space with the coefficient $s \geq 1$ and let $T: X \rightarrow X$ be a mapping. If there exist mappings $\alpha, \beta, \gamma, \delta \in \Psi$ such that for all $x, y \in X$ and for fixed $a \in X$,

$$
\begin{aligned}
\text { (a) } & \alpha(x, y, a)+\beta(x, y, a)+2 \gamma(x, y, a) \\
& +2 s \delta(x, y, a)<1 ; \\
\text { (b) } & d(T x, T y) \lesssim \alpha(x, y, a) d(x, y) \\
+ & \beta(x, y, a) \frac{[1+d(x, T x)] d(y, T y)}{1+d(x, y)} \\
+ & \gamma(x, y, a)[d(x, T x)+d(y, T y)] \\
+ & \delta(x, y, a)[d(x, T y)+d(y, T x)],
\end{aligned}
$$

then $T$ has a unique fixed point.

Proof. Let $x_{0} \in X$ and the sequence $\left\{x_{n}\right\}$ be defined by $x_{n+1}=$ $T x_{n}$, where $n=0,1,2, \ldots$ Now we show that $\left\{x_{n}\right\}$ is a Cauchy sequence. From condition (21), we have

$$
\begin{aligned}
& d\left(x_{n+1}, x_{n+2}\right)=d\left(T x_{n}, T x_{n+1}\right) \\
& \lesssim \alpha\left(x_{n}, x_{n+1}, a\right) d\left(x_{n}, x_{n+1}\right) \\
& \quad+\beta\left(x_{n}, x_{n+1}, a\right) \frac{\left[1+d\left(x_{n}, T x_{n}\right)\right] d\left(x_{n+1}, T x_{n+1}\right)}{1+d\left(x_{n}, x_{n+1}\right)} \\
& \quad+\gamma\left(x_{n}, x_{n+1}, a\right)\left[d\left(x_{n}, T x_{n}\right)+d\left(x_{n+1}, T x_{n+1}\right)\right] \\
& \quad+\delta\left(x_{n}, x_{n+1}, a\right)\left[d\left(x_{n}, T x_{n+1}\right)+d\left(x_{n+1}, T x_{n}\right)\right] \\
& \lesssim \\
& \quad \alpha\left(x_{0}, x_{0}, a\right) d\left(x_{n}, x_{n+1}\right) \\
& \quad+\beta\left(x_{0}, x_{0}, a\right) d\left(x_{n+1}, x_{n+2}\right) \\
& \quad+\gamma\left(x_{0}, x_{0}, a\right)\left[d\left(x_{n}, x_{n+1}\right)+d\left(x_{n+1}, x_{n+2}\right)\right] \\
& \quad+s \delta\left(x_{0}, x_{0}, a\right)\left[d\left(x_{n}, x_{n+1}\right)+d\left(x_{n+1}, x_{n+2}\right)\right] .
\end{aligned}
$$


Therefore,

$$
\begin{aligned}
& \left|d\left(x_{n+1}, x_{n+2}\right)\right| \leq \alpha\left(x_{0}, x_{0}, a\right)\left|d\left(x_{n}, x_{n+1}\right)\right|+\beta\left(x_{0}, x_{0}, a\right) \\
& \quad \cdot\left|d\left(x_{n+1}, x_{n+2}\right)\right|+\gamma\left(x_{0}, x_{0}, a\right)\left[\mid d\left(x_{n}, x_{n+1}\right)\right. \\
& \left.\quad+d\left(x_{n+1}, x_{n+2}\right) \mid\right]+s \delta\left(x_{0}, x_{0}, a\right)\left[\mid d\left(x_{n}, x_{n+1}\right)\right. \\
& \left.\quad+d\left(x_{n+1}, x_{n+2}\right) \mid\right] \\
& \quad \leq \frac{\alpha\left(x_{0}, x_{0}, a\right)+\gamma\left(x_{0}, x_{0}, a\right)+s \delta\left(x_{0}, x_{0}, a\right)}{1-\beta\left(x_{0}, x_{0}, a\right)-\gamma\left(x_{0}, x_{0}, a\right)-s \delta\left(x_{0}, x_{0}, a\right)} \mid d\left(x_{n},\right. \\
& \left.\quad x_{n+1}\right) \mid .
\end{aligned}
$$

Let $\mu=\left(\alpha\left(x_{0}, x_{0}, a\right)+\gamma\left(x_{0}, x_{0}, a\right)+s \delta\left(x_{0}, x_{0}, a\right)\right) /(1-$ $\left.\beta\left(x_{0}, x_{0}, a\right)-\gamma\left(x_{0}, x_{0}, a\right)-s \delta\left(x_{0}, x_{0}, a\right)\right)<1$; then,

$$
\left|d\left(x_{n+1}, x_{n+2}\right)\right| \leq \mu\left|d\left(x_{n}, x_{n+1}\right)\right| \quad \forall n=0,1,2, \ldots
$$

By Lemma 8, this sequence is Cauchy sequence in $(X, d)$. Since $X$ is complete, there exists some $u \in X$ such that $x_{n} \rightarrow u$ as $n \rightarrow \infty$. Next, we show that $u$ is a fixed point of $T$.

From (21), we have

$$
\begin{aligned}
& d(u, T u) \precsim s d\left(u, T x_{n}\right)+s d\left(T x_{n}, T u\right) \\
& \precsim s d\left(u, T x_{n}\right)+s \alpha\left(x_{n}, u, a\right) d\left(x_{n}, u\right) \\
& \quad+s \beta\left(x_{n}, u, a\right) \frac{\left[1+d\left(x_{n}, T x_{n}\right)\right] d(u, T u)}{1+d\left(x_{n}, u\right)} \\
& \quad+s \gamma\left(x_{n}, u, a\right)\left[d\left(x_{n}, T x_{n}\right)+d(u, T u)\right] \\
& \quad+s \delta\left(x_{n}, u, a\right)\left[d\left(x_{n}, T u\right)+d\left(u, T x_{n}\right)\right] \\
& \precsim s d\left(u, x_{n+1}\right)+s \alpha\left(x_{0}, u, a\right) d\left(x_{n}, u\right) \\
& +s \beta\left(x_{0}, u, a\right) \frac{\left[1+d\left(x_{n}, x_{n+1}\right)\right] d(u, T u)}{1+d\left(x_{n}, u\right)} \\
& +s \gamma\left(x_{0}, u, a\right)\left[d\left(x_{n}, x_{n+1}\right)+d(u, T u)\right] \\
& +s \delta\left(x_{0}, u, a\right)\left[d\left(x_{n}, T u\right)+d\left(u, x_{n+1}\right)\right] .
\end{aligned}
$$

This implies that

$$
\begin{aligned}
& |d(u, T u)| \\
& \leq s\left|d\left(u, x_{n+1}\right)\right|+s \alpha\left(x_{0}, u, a\right)\left|d\left(x_{n}, u\right)\right| \\
& \quad+s \beta\left(x_{0}, u, a\right) \frac{\left|1+d\left(x_{n}, x_{n+1}\right)\right||d(u, T u)|}{\left|1+d\left(x_{n}, u\right)\right|} \\
& \quad+s \gamma\left(x_{0}, u, a\right)\left|\left[d\left(x_{n}, x_{n+1}\right)+d(u, T u)\right]\right| \\
& \quad+s \delta\left(x_{0}, u, a\right)\left|\left[d\left(x_{n}, T u\right)+d\left(u, x_{n+1}\right)\right]\right|
\end{aligned}
$$

which on making $n \rightarrow \infty$ reduces to

$$
\begin{gathered}
|d(u, T u)| \leq s \beta\left(x_{0}, u, a\right)|d(u, T u)|+s \gamma\left(x_{0}, u, a\right) \\
\cdot|d(u, T u)|+s \delta\left(x_{0}, u, a\right)|d(u, T u)| \\
=\left[s \beta\left(x_{0}, u, a\right)+s \gamma\left(x_{0}, u, a\right)+s \delta\left(x_{0}, u, a\right)\right] \\
\cdot|d(u, T u)| \leq s\left[\alpha\left(x_{0}, u, a\right)+\beta\left(x_{0}, u, a\right)\right. \\
\left.+2 \gamma\left(x_{0}, u, a\right)+2 \delta\left(x_{0}, u, a\right)\right]|d(u, T u)|,
\end{gathered}
$$

a contradiction, and so $|d(u, T u)|=0$; that is, $u=T u$. This implies that $u$ is a fixed point of $T$.

Uniqueness of fixed point is an easy consequence of condition (22). This completes the proof.

Corollary 13. Let $(X, d)$ be a complete complex valued $b$ metric space with the coefficient $s \geq 1$ and let $T: X \rightarrow X$ be a mapping. If there exist mappings $\alpha, \beta, \gamma, \delta \in \Psi$ such that for all $x, y \in X$ and for some fixed $n$,

$$
\begin{aligned}
& \text { (a) } \alpha(x, y, a)+\beta(x, y, a)+2 \gamma(x, y, a) \\
& \quad+2 s \delta(x, y, a)<1 ; \\
& \text { (b) } d\left(T^{n} x, T^{n} y\right) \lesssim \alpha(x, y, a) d(x, y) \\
& +\beta(x, y, a) \frac{\left[1+d\left(x, T^{n} x\right)\right] d\left(y, T^{n} y\right)}{1+d(x, y)} \\
& +\gamma(x, y, a)\left[d\left(x, T^{n} x\right)+d\left(y, T^{n} y\right)\right] \\
& +\delta(x, y, a)\left[d\left(x, T^{n} y\right)+d\left(y, T^{n} x\right)\right],
\end{aligned}
$$

then $T$ has a unique fixed point.

Proof. By Theorem 12, there exists $v \in X$ such that $T^{n} v=v$. Then,

$$
\begin{aligned}
& d(T v, v)=d\left(T T^{n} v, T^{n} v\right)=d\left(T^{n} T v, T^{n} v\right) \\
& \lesssim \alpha(T v, v, a) d(T v, v) \\
& \quad+\beta(T v, v, a) \frac{\left[1+d\left(x, T^{n} T v\right)\right] d\left(v, T^{n} v\right)}{1+d(T v \cdot v)} \\
& \quad+\gamma(T v, v, a)\left[d\left(T v, T^{n} T v\right)+d\left(v, T^{n} v\right)\right] \\
& \quad+\delta(T v, v, a)\left[d\left(T v, T^{n} v\right)+d\left(v, T^{n} T v\right)\right] \\
& \lesssim \alpha(T v, v, a) d(T v, v)
\end{aligned}
$$

$$
\begin{aligned}
& +\beta(T v, v, a) \frac{\left[1+d\left(x, T T^{n} v\right)\right] d(v, v)}{1+d(T v \cdot v)} \\
& +\gamma(T v, v, a)\left[d\left(T v, T T^{n} v\right)+d(v, v)\right] \\
& +\delta(T v, v, a)\left[d(T v, v)+d\left(v, T T^{n} v\right)\right] \\
= & \alpha(T v, v, a) d(T v, v)+2 \delta(T v, v, a) d(T v, v) \\
= & (\alpha+2 \delta)(T v, v, a) d(T v, v)
\end{aligned}
$$

and so $d(T v, v)=0$. So $T v=v$. Therefore, the fixed point of $T$ is unique. 
Example 14. Let $X=[0,1]$ and $d: X \times X \rightarrow \mathbb{C}$ be defined by $d(x, y)=i|x-y|^{2}$ for all $x, y \in X$. Then, $(X, d)$ is a complex valued $b$-metric space with the coefficient $s=2$. Now we define self-mappings $S, T: X \rightarrow X$ by $S(x)=x / 4$ and $T(y)=$ $y / 4$. Further, for all $x, y \in X$ and for fixed $a=1 / 3 \in X$, we define the functions $\alpha, \beta, \gamma, \delta: X \times X \times X \rightarrow[0,1)$ by

$$
\begin{aligned}
& \alpha(x, y, a)=\left(\frac{x}{4}+\frac{y}{5}+a\right), \\
& \beta(x, y, a)=\frac{x y a}{10} \\
& \gamma(x, y, a)=\frac{x^{2} y^{2} a^{2}}{10} \\
& \delta(x, y, a)=\frac{x^{3} y^{3} a^{3}}{10} .
\end{aligned}
$$

Clearly $\alpha(x, y, a)+\beta(x, y, a)+2 \gamma(x, y, a)+2 s \delta(x, y, a)<1$ for all $x, y \in X$ and for a fixed $a=1 / 3 \in X$.

Now consider

$$
\begin{aligned}
\alpha(T S x, y, a) & =\alpha\left(T\left(\frac{x}{4}\right), y, a\right)=\alpha\left(\frac{x}{16}, y, a\right) \\
& =\frac{x}{64}+\frac{y}{5}+a \leq \frac{x}{4}+\frac{y}{5}+a \\
& =\alpha(x, y, a) .
\end{aligned}
$$

That is, $\alpha(T S x, y, a) \leq \alpha(x, y, a)$ for all $x, y \in X$ and for a fixed $a=1 / 3 \in X$. Now

$$
\begin{aligned}
\alpha(x, \text { ST } y, a) & =\alpha\left(x, S\left(\frac{y}{4}\right), a\right)=\alpha\left(x, \frac{y}{16}, a\right) \\
& =\frac{x}{4}+\frac{y}{80}+a \leq \frac{x}{4}+\frac{y}{5}+a \\
& =\alpha(x, y, a) .
\end{aligned}
$$

That is, $\alpha(x, S T y, a) \leq \alpha(x, y, a)$ for all $x, y \in X$ and for a fixed $a=1 / 3 \in X$. Similarly, we can show that

$$
\begin{aligned}
& \beta(T S x, y, a) \leq \beta(x, y, a), \\
& \beta(x, S T y, a) \leq \beta(x, y, a), \\
& \gamma(T S x, y, a) \leq \gamma(x, y, a), \\
& \gamma(x, S T y, a) \leq \gamma(x, y, a), \\
& \delta(T S x, y, a) \leq \delta(x, y, a), \\
& \delta(x, S T y, a) \leq \delta(x, y, a) .
\end{aligned}
$$

Now for the verification of inequality (5), it is sufficient to show that $(S x, T y) \lesssim \alpha(x, y, a) d(x, y)$.
Consider

$$
\begin{aligned}
d(S x, T y)= & d\left(\frac{x}{4}, \frac{y}{4}\right)=\left|\frac{x}{4}-\frac{y}{4}\right|^{2} i=\frac{1}{16}|x-y|^{2} i \\
\lesssim & \frac{1}{3}|x-y|^{2} i \precsim\left(\frac{x}{4}+\frac{y}{5}+\frac{1}{3}\right)|x-y|^{2} i \\
= & \alpha(x, y, a) d(x, y), \\
& \forall x, y \in X, \text { for } a=\frac{1}{3} \in X .
\end{aligned}
$$

That is, $d(S x, T y) \precsim \alpha(x, y, a) d(x, y)$ for all $x, y \in X$ and for fixed $a=1 / 3 \in X$. Therefore, all the conditions of Theorem 9 are satisfied, also $x=0$ remains fixed under $S$ and $T$ and is indeed unique.

\section{Competing Interests}

The authors declare that they have no competing interests.

\section{References}

[1] A. Azam, B. Fisher, and M. Khan, "Common fixed point theorems in complex valued metric spaces," Numerical Functional Analysis and Optimization, vol. 32, no. 3, pp. 243-253, 2011.

[2] C. Klin-eam and C. Suanoom, "Some common fixed-point theorems for generalized-contractive-type mappings on complexvalued metric spaces," Abstract and Applied Analysis, vol. 2013, Article ID 604215, 6 pages, 2013.

[3] M. A. Kutbi, A. Azam, J. Ahmad, and C. Di Bari, "Some common coupled fixed point results for generalized contraction in complex-valued metric spaces," Journal of Applied Mathematics, vol. 2013, Article ID 352927, 10 pages, 2013.

[4] H. K. Nashine, M. Imdad, and M. Hasan, "Common fixed point theorems under rational contractions in complex valued metric spaces," Journal of Nonlinear Science and Its Applications, vol. 7, no. 1, pp. 42-50, 2014.

[5] H. K. Nashine and B. Fisher, "Common fixed point theorems for generalized contraction involving rational expressions in complex valued metric spaces," Analele Stiintifice ale Universitatii Ovidius Constanta, vol. 23, no. 2, pp. 179-185, 2015.

[6] F. Rouzkard and M. Imdad, "Some common fixed point theorems on complex valued metric spaces," Computers \& Mathematics with Applications, vol. 64, no. 6, pp. 1866-1874, 2012.

[7] N. Singh, D. Singh, A. Badal, and V. Joshi, "Fixed point theorems in complex valued metric spaces," Journal of the Egyptian Mathematical Society, vol. 24, no. 3, pp. 402-409, 2016.

[8] W. Sintunavarat and P. Kumam, "Generalized common fixed point theorems in complex valued metric spaces and applications," Journal of Inequalities and Applications, vol. 2012, article 84, 12 pages, 2012.

[9] W. Sintunavarat, Y. J. Cho, and P. Kumam, "Urysohn integral equations approach by common fixed points in complex-valued metric spaces," Advances in Difference Equations, vol. 2013, article 49, 14 pages, 2013.

[10] K. Sitthikul and S. Saejung, "Some fixed point theorems in complex valued metric spaces," Fixed Point Theory and Applications, vol. 2012, article 189, 11 pages, 2012.

[11] K. P. R. Rao, P. R. Swamy, and J. R. Prasad, "A common fixed point theorem in complex valued b-metric spaces," Bulletin of Mathematics and Statistics Research, vol. 1, no. 1, pp. 1-8, 2013. 
[12] A. K. Dubey, R. Shukla, and R. P. Dubey, "Some fixed point theorems in complex valued b-metric spaces," Journal of Complex Systems, vol. 2015, Article ID 832467, 7 pages, 2015.

[13] A. K. Dubey, "Common fixed point results for contractive mappings in complex valued b-metric spaces," Nonlinear Functional Analysis and Applications, vol. 20, no. 2, pp. 257-268, 2015.

[14] A. K. Dubey, R. Shukla, and R. P. Dubey, "Some common fixed point theorems for contractive mappings in complex valued bmetric spaces," Asian Journal of Mathematics and Apllications, vol. 2015, Article ID ama0266, 13 pages, 2015.

[15] A. A. Mukheimer, "Some common fixed point theorems in complex valued $b$-metric spaces," The Scientific World Journal, vol. 2014, Article ID 587825, 6 pages, 2014.

[16] B. K. Dass and S. Gupta, "An extension of Banach contraction principle through rational expression," Indian Journal of Pure and Applied Mathematics, vol. 6, no. 12, pp. 1455-1458, 1975. 


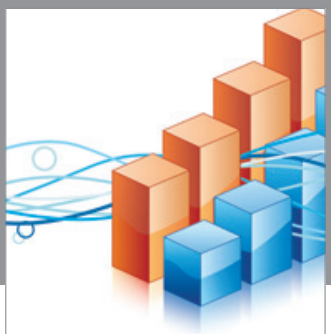

Advances in

Operations Research

vatem alat4

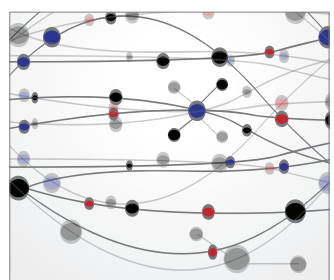

\section{The Scientific} World Journal
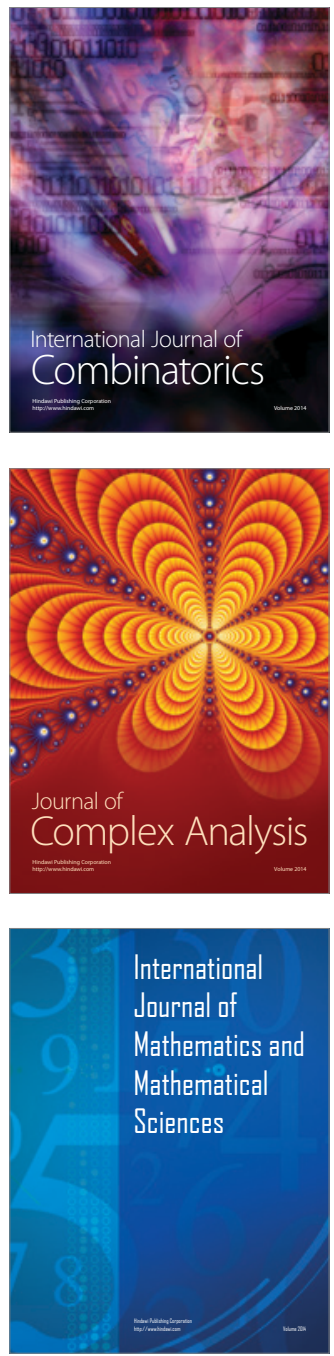
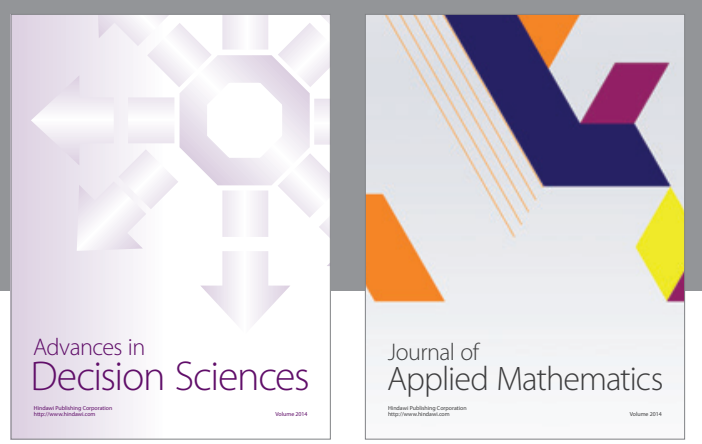

Algebra

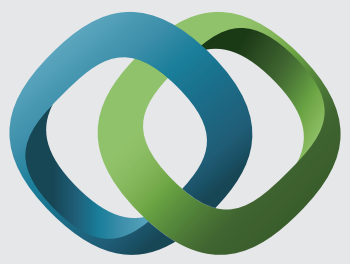

\section{Hindawi}

Submit your manuscripts at

http://www.hindawi.com
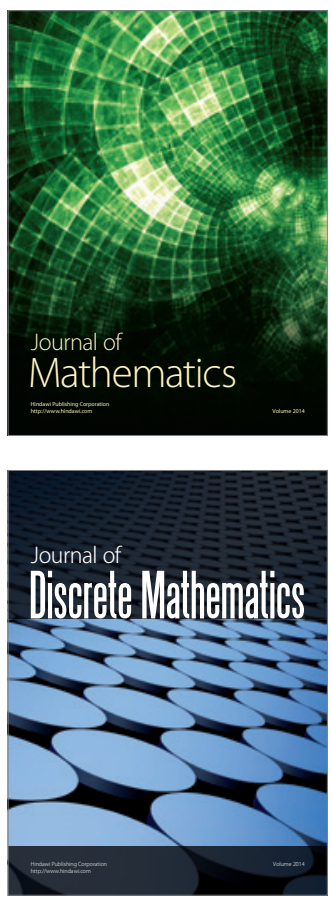

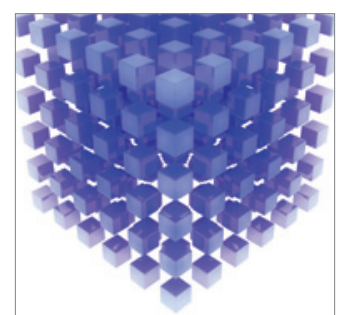

Mathematical Problems in Engineering
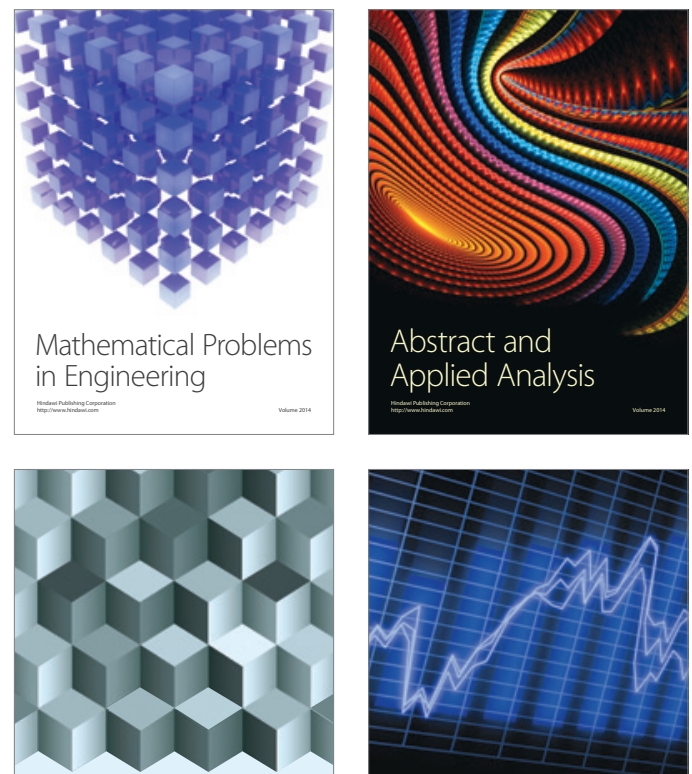

Journal of

Function Spaces

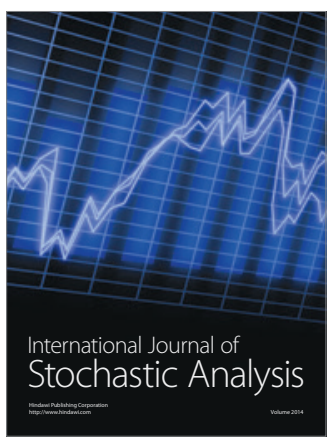

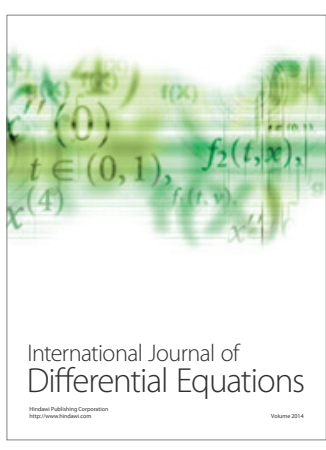
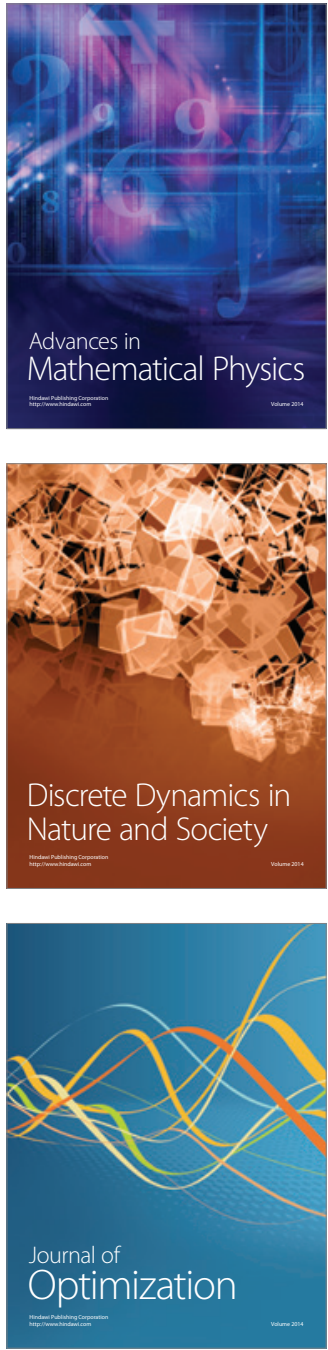